\title{
An Investigation of Effects and Safety of Pipelines due to Twin Tunneling
}

\author{
Shao Yu, ${ }^{1,2}$ Riyan Lan, ${ }^{2}$ Junhui Luo $\mathbb{D}^{1,2}$ Zhibo Duan ${ }^{1}{ }^{3}$ and Shaokun Ma $\mathbb{D}^{3}$ \\ ${ }^{1}$ Guangxi Communications Design Group Co., Ltd., Nanning 530029, China \\ ${ }^{2}$ Guangxi Xinfazhan Communication Group Co., Ltd., Nanning 530029, China \\ ${ }^{3}$ College of Civil Engineering and Architecture, Guangxi University, Nanning 530004, China
}

Correspondence should be addressed to Zhibo Duan; 752490978@qq.com and Shaokun Ma; mashaokun@sina.com

Received 3 December 2020; Revised 11 March 2021; Accepted 11 May 2021; Published 28 May 2021

Academic Editor: Loke Foong

Copyright ( $\odot 2021$ Shao Yu et al. This is an open access article distributed under the Creative Commons Attribution License, which permits unrestricted use, distribution, and reproduction in any medium, provided the original work is properly cited.

\begin{abstract}
To efficiently and accurately predict the effects of twin tunneling on adjacent buried pipelines, the effects of upward and downward relative pipeline-soil interactions were considered. A series of numerical parametric studies encompassing 8640 conditions were performed to investigate the responses of a pipeline to twin tunneling. Based on the dimensionless analysis and normalized calculation results, the concept of equivalent relative pipeline-soil stiffness was proposed. Additionally, expressions for the relative pipeline-soil stiffness and relative pipeline curvature and for the relative pipeline-soil stiffness and relative pipeline settlement were established, along with the related calculation plots. Relying on a comparison of prediction results, centrifuge model test results, and field measured results, the accuracy and reliability of the obtained expressions for predicting the bending strain and settlement of adjacent buried pipelines caused by twin tunneling were validated. Based on the calculation method, the maximum bending strain and maximum settlement of pipelines can be calculated precisely when the pipeline parameters, burial depth, soil parameters, and curve parameters of ground settlement due to tunneling are provided. The proposed expressions can be used not only to predict the maximum bending strain and maximum settlement of pipelines caused by single and twin tunneling but also to evaluate the effects of single and twin tunneling on the safety of existing buried pipelines. The relevant conclusions of this article can also provide a theoretical basis for the normal service of buried pipelines adjacent to subway tunnels.
\end{abstract}

\section{Introduction}

Due to stratum displacement induced by shield construction, additional stress and displacement are inevitably produced in adjacent existing buried pipelines, which further affect the normal use of such pipelines, or even leads to their failure or burst [1]. The issue of tunnel-pipeline-soil interaction is receiving increasingly close attention and is being widely explored by geotechnical scholars in many systematic and meaningful studies $[2,3]$.

Following the theory of continuum elasticity, a new foundation model established by Klar et al. [4] was used to analyze the issue of tunnel-pipeline-soil interaction and was compared with the theory used by Attewell et al. [5]. Yu et al. [6] further explored this problem, established a foundation model more suitable for the analysis of this issue, and compared its results with the results of Klar et al. [4] using the theory of continuum elasticity. Recently, Klar and Marshall [7] adopted the theory of continuum elasticity to demonstrate that the volume loss deduced from the ground settlement under the greenfield condition was equal to that deduced from pipeline deformation in the presence of pipelines, and put forward a simple expression for predicting the bending moment of pipelines. Multiple tunnel-pipelinesoil interaction conditions were simulated by Wang et al. [8] and Shi and Chen [9], and an expression for calculating the maximum bending strain of pipelines caused by single tunneling was developed. To consider the effect of soil high nonlinearity, a linear-equivalent approach was proposed by Vorster et al. [10]. The law of relative pipeline-soil stiffness varying nonlinearly with soil strain was established based on the relationship for soil stiffness and strain around pipelines. 
However, their results were too conservative. Based on the equivalent soil shear strain proposed by Marshall et al. [11], Klar et al. [12] solved the difficult problem of determining relative pipeline-soil displacement and offered the procedure for calculating the maximum bending moment of pipelines caused by tunneling by considering soil nonlinearity. Based on the Pasternak model and a uniform ground movement model, an energy solution for predicting buried pipeline response induced by tunneling was proposed by Shi et al. [13]. Multiple research methods were applied by Zhang et al. [14], who investigated the interaction mechanism between an existing pipeline and quasirectangular tunneling in clays. An improved Winkler modulus was proposed by Huang et al. [15] for the analysis of the response of jointed pipelines to tunneling. Saiyar et al. [16] investigated the effects of stratum displacement on pipelines of varying stiffness. During the investigation, it was pointed out that the analysis of pipeline-soil interaction should give reasonable consideration to soil nonlinearity, as the results obtained in this manner would be more pragmatic than the results acquired through modifying the ground settlement mode only.

In the above examples, scholars only focused on the effects of single tunneling on existing buried pipelines, but rarely considered the case of twin tunneling. Klar and Marshall [7] and Wei et al. [17] mentioned that the superposition principle could be used to analyze the effects of twin tunneling on existing adjacent buried pipelines. Nevertheless, the analysis by Ma et al. $[18,19]$ revealed that, due to its inability to reasonably consider the effects of subsequent tunneling on relative pipeline-soil stiffness, the superposition principle would produce significant differences between predicted and test results. To study pipeline-soil interactions, a series of numerical parametric analyses were performed by adopting the curvilinear equation proposed by Vorster et al. [10] for describing the ground surface settlement caused by tunneling. On this basis, the calculation formulas and design charts which can be used to predict the maximum bending strain and maximum settlement of pipelines caused by single and twin tunneling were proposed. The results obtained by the proposed method are more accurate than those by other methods and can be used to study the influence of multi tunnel excavation on nearby buried pipelines.

\section{Distribution Curve of Ground Settlement Caused by Twin Tunneling}

Vorster et al. [10] proposed adopting the following modified Gaussian distribution curve to describe the ground settlement caused by tunneling:

$$
\begin{aligned}
S_{v}(x) & =\frac{n S_{\max }}{(n-1)+\exp \left[\left(\alpha\left(x^{2} / i^{2}\right)\right)\right]}, \\
n & =\exp (\alpha) \frac{2 \alpha-1}{2 \alpha+1}+1,
\end{aligned}
$$

where $S_{v}(x)$ is the vertical displacement of soil (greenfield condition); $S_{\text {max }}$ is the maximum vertical surface settlement value; $i$ is the distance from any point to the ground surface settlement inflection point; and $n$ and $\alpha$ are shape parameters of the soil settlement (equation 1 degrades into a Gaussian distribution curve when $\alpha=0.5$ (i.e., $n=1$ )).

Marshall et al. [20] used four empirical equations for ground surface settlement to fit the ground surface settlement measured in the field and obtained by centrifuge model tests and found that the modified Gaussian distribution curve could better fit the data than the other three empirical curves. Klar and Marshall [7] discovered that the modified Gaussian distribution curve could well fit a series of superposed Gaussian distribution curves (equation 3, Figure 1). It can be seen from Figure 1 that the modified Gaussian distribution curve can be used to fit the surface settlement curve caused by twin tunneling. On the basis of analyzing measured field data, Suwansawat and Einstein [21] pointed out that the ground surface settlement curve caused by twin tunneling could be put through superposed fitting with two groups of Gaussian distribution curves. Therefore, the modified Gaussian distribution curve can be used to satisfactorily describe the ground surface settlement caused by twin tunneling.

$$
S_{v}(x)=\sum_{k=1}^{2} s_{\max , k} \exp \left(-\frac{1}{2} \frac{x^{2}}{i_{k}^{2}}\right)=\frac{V_{s, 1}}{\sqrt{2 \pi} i_{1}} \exp \left(-\frac{1}{2} \frac{x^{2}}{i_{1}^{2}}\right)+\frac{V_{s, 2}}{\sqrt{2 \pi} i_{2}} \exp \left(-\frac{1}{2} \frac{x^{2}}{i_{2}^{2}}\right)
$$

\section{Pipeline-Soil Interaction Mechanism}

Relative pipeline-soil displacement is the root cause of the different pipeline-soil interaction mechanisms in different longitudinal zones of a pipeline. With the aid of centrifuge tests, Vorster [22] and Marshall [23] claimed that the pipeline-soil interaction mechanism caused by tunneling primarily involved the following five zones: (1) the zone unaffected by the pipeline, (2) the pipeline-soil detachment zone, (3) the downward compression zone, (4) the pulldown zone, and (5) the stretching zone. In practical engineering, the hyperbolic model shown in Figure 2 (the dotted line) is commonly used to describe the vertical pipeline-soil interaction. The hyperbolic model shown in Figure 3 (the dotted line) describes the axial pipeline-soil interaction. For ease of use, they are simplified to a combination of an elastic model and an ideal elastoplastic model (the solid lines in Figures 2 and 3). 


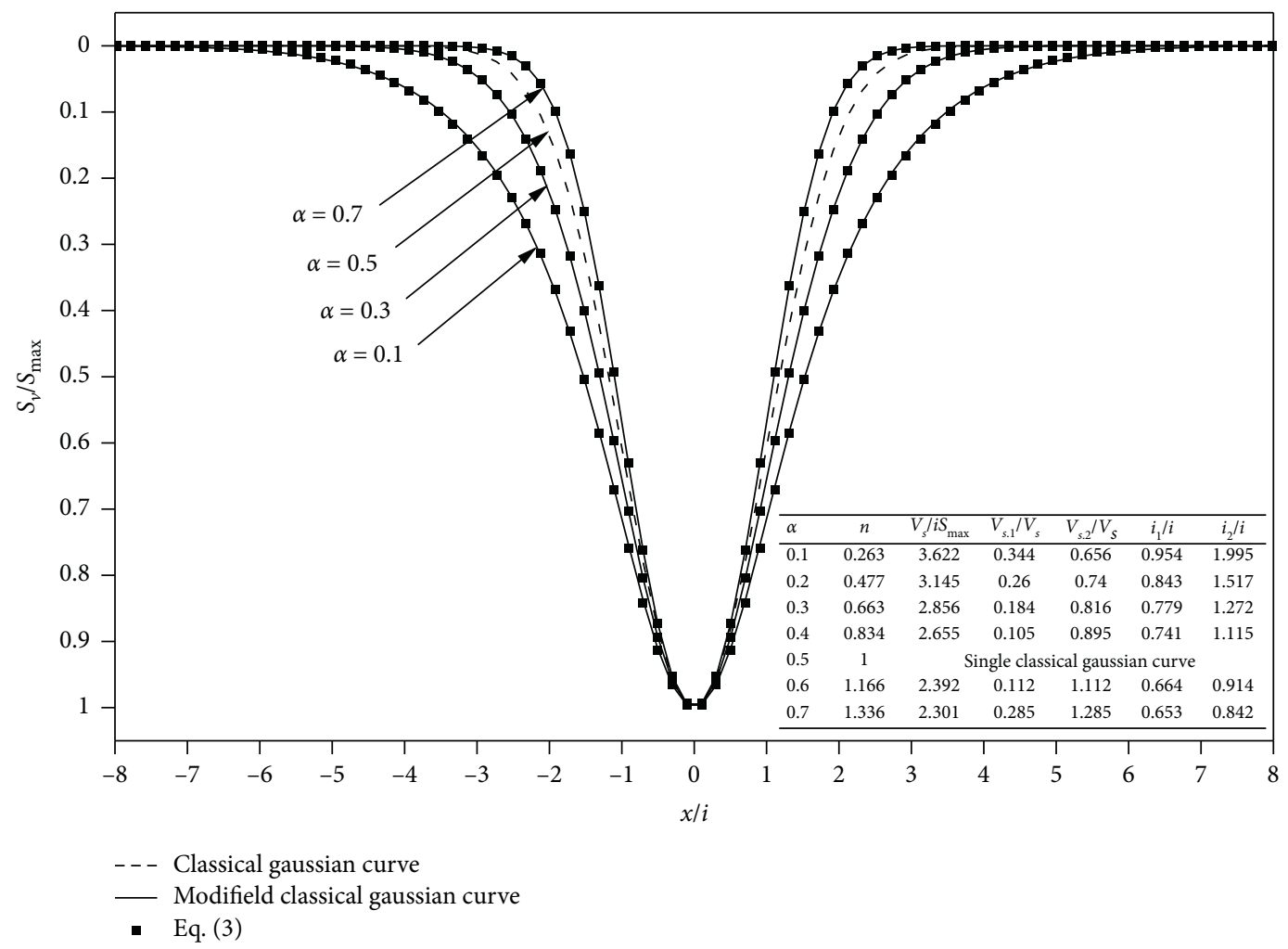

Figure 1: Modified Gaussian and fitted 2-function Gaussian curves.

In Figure 2, $q_{u}$ and $q_{d}$ denote the maximum resistance forces of soil when the relative pipeline-soil displacement is upward and downward, respectively. Their magnitudes are closely related to the magnitude of the relative pipeline-soil displacement and are expressed as follows:

$$
\begin{aligned}
q_{u} & =N_{v} \gamma H D, \\
q_{d} & =\gamma H N_{q} D+\frac{1}{2} \gamma D^{2} N_{\gamma},
\end{aligned}
$$

where $N_{v}$ denotes dimensionless soil resistance coefficient and $N_{q}$ and $N_{\gamma}$ denote the basic bearing capacity factors of strip foundation. $N_{v}, N_{q}$, and $N_{\gamma}$ can be calculated by the following formulas:

$$
\begin{aligned}
& N_{v}=\left(\frac{\varphi H}{44 D}\right) \leq N_{q}, \\
& N_{q}=\exp (\pi \tan \varphi) \tan ^{2}\left(45+\frac{\varphi}{2}\right), \\
& N_{\gamma}=\exp (0.18 \varphi-2.5),
\end{aligned}
$$

where $\gamma$ denotes the bulk unit weight of soil; $H$ denotes the distance from ground surface to pipeline center; $D$ denotes pipeline diameter; and $\varphi$ denotes the peak internal friction angle of soil.

In Figure 2, $\delta_{r u}$ and $\delta_{r d}$ denote the relative pipeline-soil displacement thresholds. That is, when the upward and downward relative pipeline-soil displacements exceed $\delta_{r u}$ and $\delta_{r d}$, the resistance forces of soil will reach $q_{u}$ and $q_{d}$, respectively. When soil compaction is in the loose to dense sand range, $\delta_{r u}$ and $\delta_{r d}$ range from 0.005 to $0.015 \mathrm{H}$ and 0.1 to $0.15 D$, respectively [24]. In Figure $3, q_{a}$ denotes the maximum resistance force of the soil when the axial relative pipeline-soil displacement reaches $\delta_{r a}$. When the pipe is buried in sand, $\delta_{r a}$ ranges from 2.5 to $5.0 \mathrm{~mm} \mathrm{[24].} \mathrm{The} \mathrm{value}$ of $q_{a}$ can be calculated by using the following equation:

$$
q_{a}=\frac{1}{2} \pi D \gamma H\left(1+K_{0}\right) \tan \delta
$$

where $K_{0}$ denotes the coefficient of Earth pressure at rest and $\delta$ denotes the internal friction angle of the pipeline-soil contact surface.

\section{Calculation Method and Verification}

4.1. Calculation Method. Figure 4 shows the schematic diagrams of vertical (a) and axial (b) pipeline-soil interaction. Their interactive relationships are both embedded in the Pipeline Soil Interaction (PSI) unit of ABAQUS software. In the calculation process, the vertical pipeline-soil interaction and the axial pipeline-soil interaction are adopted according to current relative pipeline-soil displacement. Internal forces and deformations generated by external loads on the pipeline are described using beam elements. The DISP subroutine is programmed according to equation (1). In the calculation, the ground surface settlement caused by tunneling is applied on ground surface as a distributed additional displacement, as shown in Figure 4(a). 


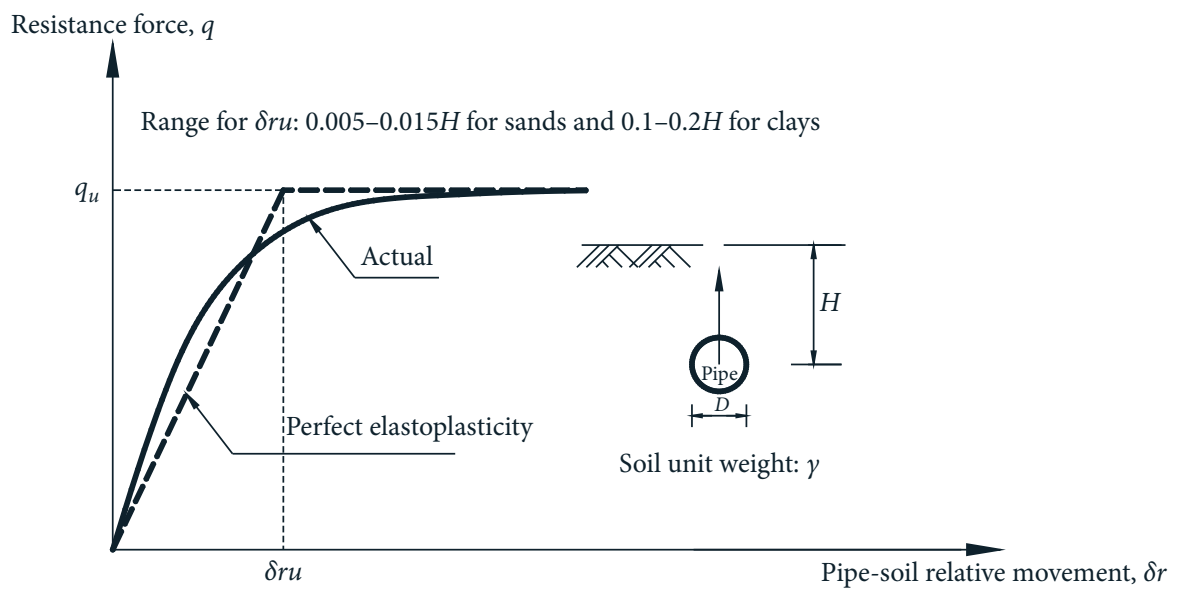

(a)

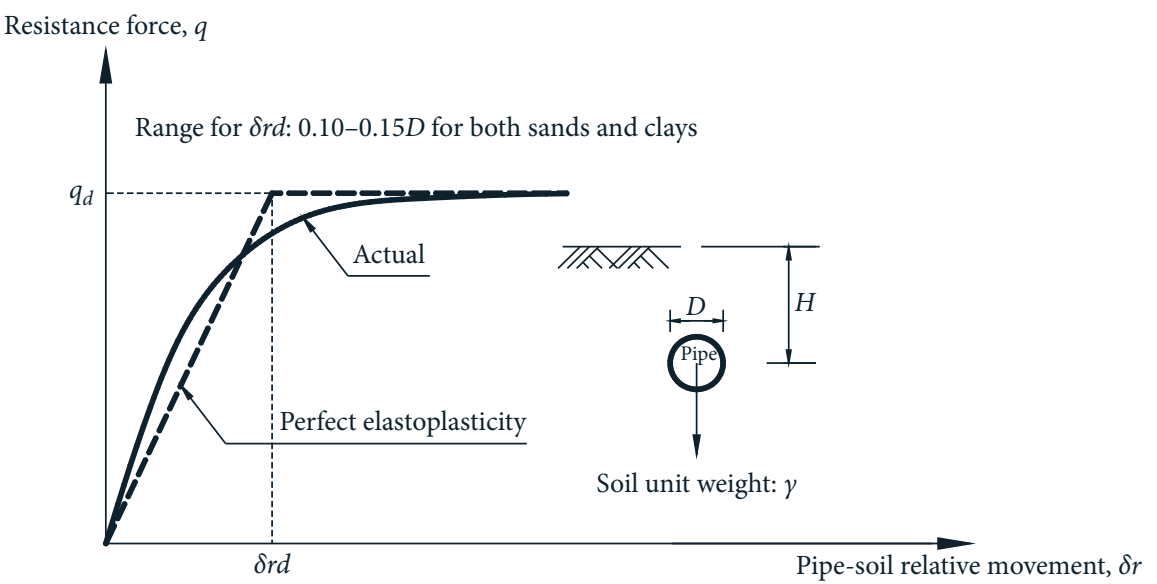

(b)

FIGURE 2: Force-displacement response of pipeline-soil interaction: (a) upward relative displacement; (b) downward relative displacement $[24,25]$.

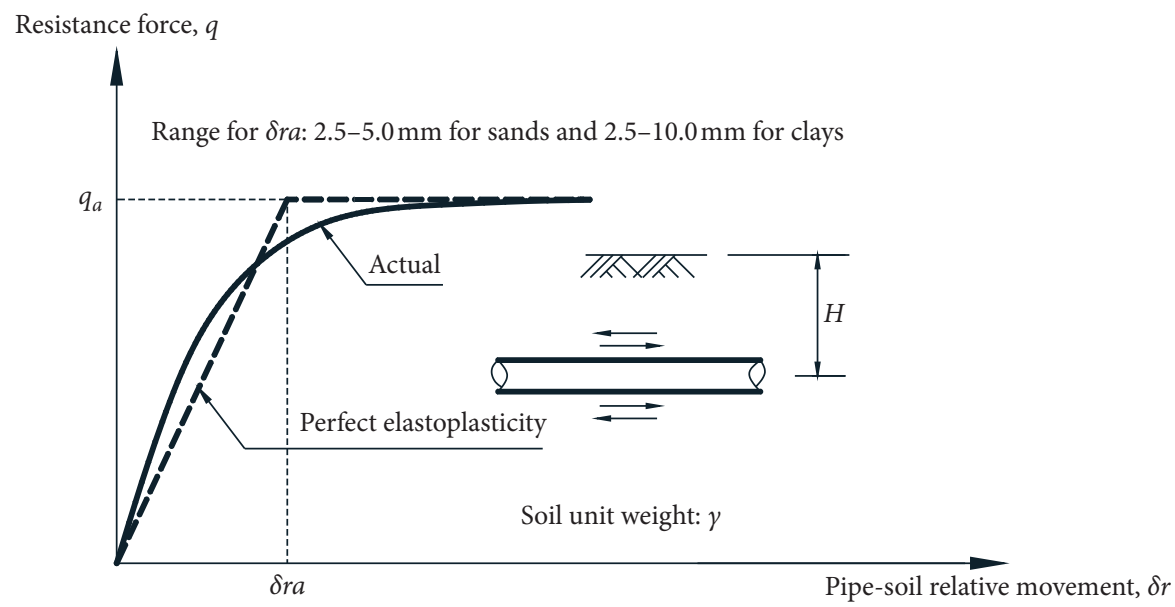

Figure 3: Force-axial relative displacement response of pipeline-soil interaction [24, 25].

4.2. Verification of the Calculation Method. A full-scale field test was carried out by Takagi et al. [25] to investigate the effect on a steel pipeline due to a single tunneling with a diameter of $4.84 \mathrm{~m}$. In the test, the tunnel burial depth was
$8.35 \mathrm{~m}$, and the length, diameter, and wall thickness of the pipeline were $13.5 \mathrm{~m}, 0.165 \mathrm{~m}$, and $5 \mathrm{~mm}$, respectively. The elastic modulus of the pipeline was $210 \mathrm{GPa}$, and Poisson's ratio was 0.3 . The distance from ground surface to the top of 


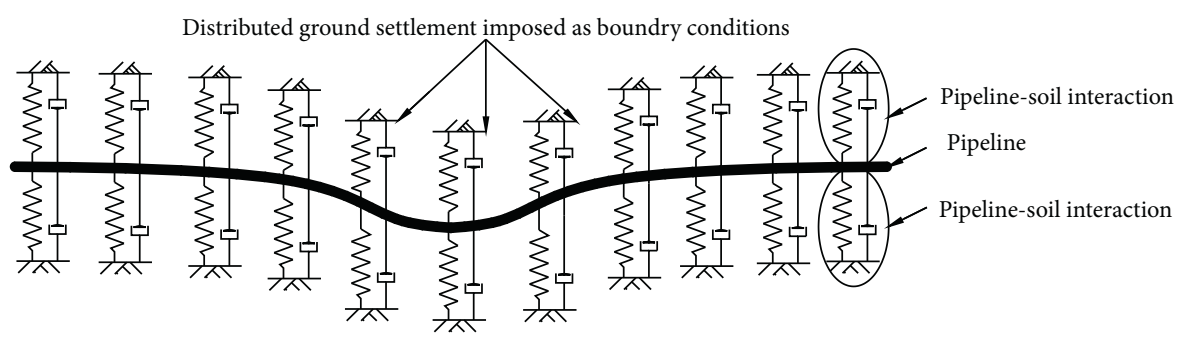

(a)

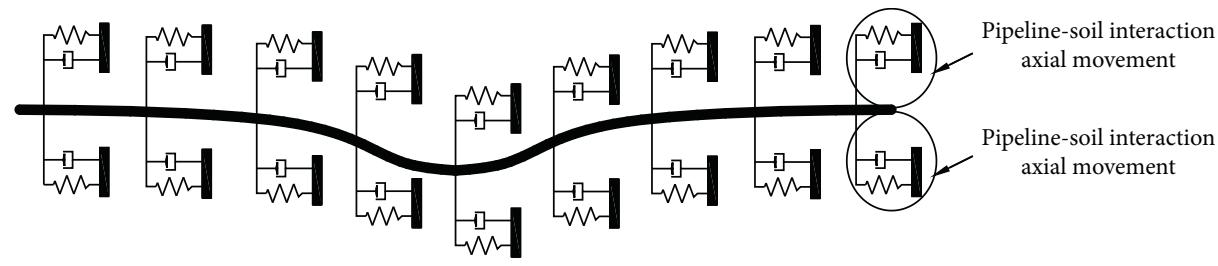

(b)

FIGURE 4: Schematic diagram of pipeline-soil interaction: (a) upward and downward relative displacements; (b) axial relative displacement.

the pipeline was $1.5 \mathrm{~m}$; i.e., $H=1.58 \mathrm{~m}$. The pipeline was vertical to the tunnel, and the distances from the tunnel axis to the two ends of the pipeline were $7.3 \mathrm{~m}$ and $6.2 \mathrm{~m}$, respectively. $q_{u}$ and $q_{d}$ were $45.4 \mathrm{kN} / \mathrm{m}$ and $364.6 \mathrm{kN} / \mathrm{m}$, respectively. $\delta_{r u}$ and $\delta_{r d}$ were $0.0158 \mathrm{~m}$ and $0.0165 \mathrm{~m}$, respectively. Equation (1) was employed to fit field measured ground settlement (Figure 5), and the fitted curve matched well with measured data $\left(S_{\max }=0.04785 \mathrm{~m}, i=1.6896 \mathrm{~m}\right.$, $\alpha=0.08818$ ). Because the pipeline stiffness is greater than the soil stiffness, the calculated maximum pipeline settlement was slightly smaller than ground surface settlement (Figure 5). Therefore, relative pipeline-soil stiffness was low under this operating condition. Furthermore, at the site of maximum pipeline settlement, the pipeline showed an upward relative displacement relative to the soil. The pipeline bending strain caused by tunneling was also measured by Takagi et al. [25]. Given that the distances from the tunnel axis to the two ends of the pipeline were unequal, the measured and calculated pipeline bending strains were asymmetric (Figure 6).

\section{Parametric Analysis}

When adopting the above method, reasonable pipeline settlement and bending strain due to tunneling can be obtained with the following parameters only: curve parameters of ground settlement caused by tunneling $\left(S_{\max }, i\right.$, and $\alpha$ ), pipeline dimension parameters (diameter $D$ and wall thickness $T$ ), pipeline material parameters ( $E$ and $\nu$ ), cover depth of the pipeline $(C)$, and soil parameters $(\gamma, \varphi)$. The common value ranges of the above parameters in practical engineering are selected in this section for 8640 individual calculations in order to identify the effects of tunneling on existing buried pipelines under different combination operating conditions.

The value ranges of the parameters of the ground settlement curve $\left(S_{\max }, i\right.$, and $\left.\alpha\right)$ used in this calculation were $0.025-0.075 \mathrm{~m}, 6-15 \mathrm{~m}$, and $0.1-1.8$, respectively, covering all operating conditions of ground surface settlement caused by single tunneling and adjacent twin tunneling in current urban subway engineering. Three materials, i.e., iron, cast iron, and PVC, were used in the pipelines, with elastic moduli of 210,84 , and $3.3 \mathrm{GPa}$, respectively. Table 1 lists the pipeline dimension parameters used in the calculation, which basically covered all pipeline dimensions commonly used in municipal engineering. Three thickness values $(C)$ were introduced for the pipeline cover depth, i.e., 1.2, 2.4, and $4.0 \mathrm{~m}$. Table 2 lists the soil parameters of three different compactness selected in calculation.

\section{Dimensionless Analysis of the Pipeline Responses due to Tunneling}

The consideration of the current relative pipeline-soil stiffness is of vital significance for accurately assessing the internal forces and deformations of pipelines [23]. This study aims to predict the effects of twin tunneling on buried pipelines more accurately. Based on the relative pipeline-soil stiffness proposed by Wang et al. [8], the effects of $\alpha$ and $n$ in equation (1) on relative pipeline-soil stiffness are considered, and a new relative pipeline-soil stiffness is put forward. The new relative pipeline-soil stiffness can be expressed as follows:

$$
R=\frac{E_{p} I_{p}}{K_{u}^{0.9} K_{d}^{0.1}[(\sqrt{(n / 2 \alpha)}) i]^{4}} \cdot\left(\frac{S_{\max }}{(\sqrt{(n / 2 \alpha)}) i}\right)^{0.25} .
$$

Equation (7) can be used to calculate the relative pipeline-soil stiffness under all conditions. Compared with the relative pipe-soil stiffness expression proposed by Wang et al. [8], which can only be used to calculate the relative pipeline-soil stiffness under single tunnel excavation conditions, equation (7) can be used to calculate pipeline-soil relative stiffness under multiple tunnel excavation conditions. where $K_{u}$ and $K_{d}$, respectively, denote the equivalent moduli of soil upon upward and downward relative 
pipeline-soil displacements, which can be calculated from the following equation:

$$
\begin{aligned}
& K_{u}=\frac{q_{u}}{\delta_{r u}}, \\
& K_{d}=\frac{q_{d}}{\delta_{r d}} .
\end{aligned}
$$

The expression of the relative pipeline-soil curvature is defined as $\kappa_{p \max } / k_{g \text { max }}$, where $\kappa_{p \max }$ denotes the maximum pipeline curvature, as follows:

$$
\kappa_{p \max }=\frac{M_{\max }}{E_{p} I_{p}}
$$

where $M_{\max }$ denotes the maximum bending moment of pipelines and $E_{p} I_{p}$ denotes pipeline bending stiffness. By taking the second derivative of equation (1), the following equation can be obtained:

$$
\kappa_{\mathrm{g} \max }=\frac{\mathrm{d}^{2} S_{v}(x)}{d x^{2}}=\frac{2 n}{\left[(n-1)+\exp \left(\alpha\left(x^{2} / i^{2}\right)\right)\right]^{2}} \cdot \frac{\alpha S_{\max }\left(\alpha\left(x^{2} / i^{2}\right)\right)}{i^{2}} \cdot\left[\frac{-4 \alpha\left(x^{2} / i^{2}\right)}{(n-1)+\exp \left(\alpha\left(x^{2} / i^{2}\right)\right)}-2 \alpha \frac{x^{2}}{i^{2}}-1\right]
$$

When $x=0, \kappa_{g \max }=(2 \alpha / n) \cdot\left(S_{\max } / i^{2}\right)$, in which case $\kappa_{p \max } / k_{g \max }$ can be expressed as follows:

$$
\frac{\kappa_{p \max }}{\kappa_{g \max }}=\frac{\varepsilon_{p \max } n i^{2}}{D \alpha S_{\max }}
$$

Figure 7 provides the distribution statistics of the ratio of maximum relative pipeline-soil displacement to $\delta_{\text {ru }}$ for a total of 8640 parameter combinations. Under $40 \%$ of calculation conditions, soil responses entered the plastic stage (Figure 7). Thus, the calculation conditions have satisfactorily covered both purely elastic cases and cases with different amounts of plastic pipeline-soil relative displacement.

Figure 8 shows the relationship between the relative pipeline-soil stiffness and the relative pipeline-soil curvature. When relative pipeline-soil stiffness is lower than $10^{-4}$, the mechanical behavior of pipelines is similar to that of flexiblejointed pipelines. In other words, pipeline deformation is very close to soil deformation. With increasing relative pipeline-soil stiffness, the mechanical behavior of pipelines is stiffened. The proposed expressions for the relative pipeline-soil stiffness and relative curvature are reasonable and can be satisfactorily used for the normalization of calculation results (Figure 8). A fitting analysis was conducted on all the calculation results, and the equation for the red solid line in Figure 8 is obtained with a $R^{2}$ value of 0.986 and is expressed as follows:

$$
y=\frac{1}{12.49 x^{0.73}+1.02},
$$

where $x$ denotes relative pipeline-soil stiffness; $y=\kappa_{\text {pmax }} /$ $k_{g \text { max }}$. Figure 8 also shows the fitting curve with a confidence interval of $\pm 90 \%$, as follows:

Upper limit (the green solid line):

$$
y=\frac{1}{5.78 x^{0.73}+1.02}
$$

Lower limit (the blue solid line):

$$
y=\frac{1}{26.11 x^{0.73}+1.02} .
$$

Figure 9 shows the relationship between the relative pipeline-soil stiffness and the ratio of maximum pipeline settlement to maximum ground surface settlement. When relative pipeline-soil stiffness is lower than $10^{-2}$, the maximum pipeline settlement is almost equal to the maximum ground surface settlement. With increasing relative pipelinesoil stiffness, the maximum pipeline settlement gradually drops below the maximum ground surface settlement. This variation law is similar to the trend of the relative pipelinesoil curvature varying with relative pipeline-soil stiffness. Similarly, by adopting the regression analysis method to fit all the calculation results, the red solid line in Figure 9 is obtained with a $R^{2}$ value of 0.981 , which is expressed as follows:

$$
y=\frac{1}{2.09 x^{0.74}+1.0}
$$

where $x$ denotes relative pipeline-soil stiffness; $y=S_{p \max } /$ $S_{g \text { max }}$. Figure 9 also shows the fitting curve with a confidence interval of $\pm 90 \%$, as expressed below:

Upper limit (the green solid line):

$$
y=\frac{1}{0.98 x^{0.65}+1.0},
$$

Lower limit (the blue solid line):

$$
y=\frac{1}{5.18 x^{0.84}+1.0} .
$$

\section{Verification Example}

To investigate the effects of twin tunneling on adjacent existing buried pipelines under different layouts, Ma et al. $[18,19]$ conducted four groups of centrifuge model tests. In Test 1 and Test 2, the two tunnels had the same burial depth, 


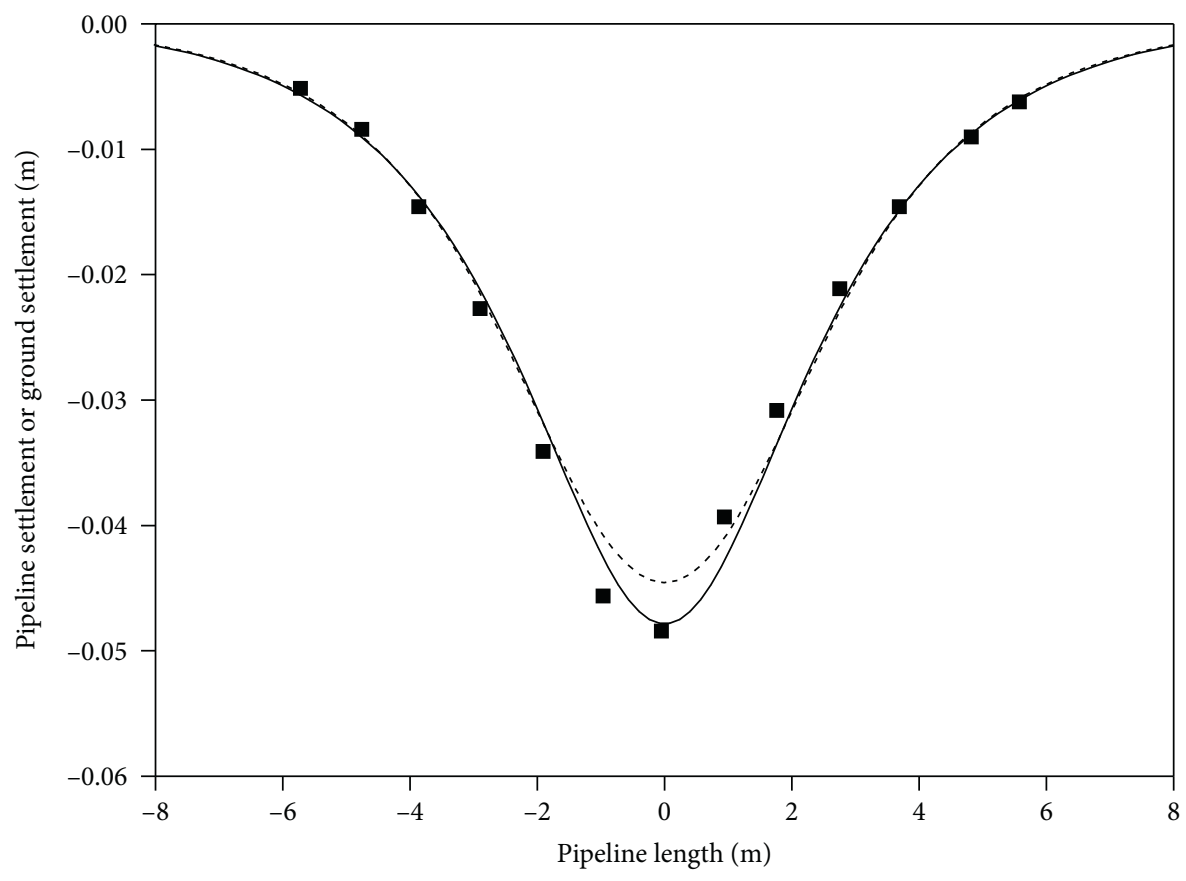

- Observed ground settlement [25]

Fitted by modified gaussian curve $\left(S_{\max }=0.04785 \mathrm{~m}, i=1.6896 \mathrm{~m}, \alpha=0.08818\right)$

- - - Pipeline settlement from computation

FIgURE 5: Pipeline and ground settlements.

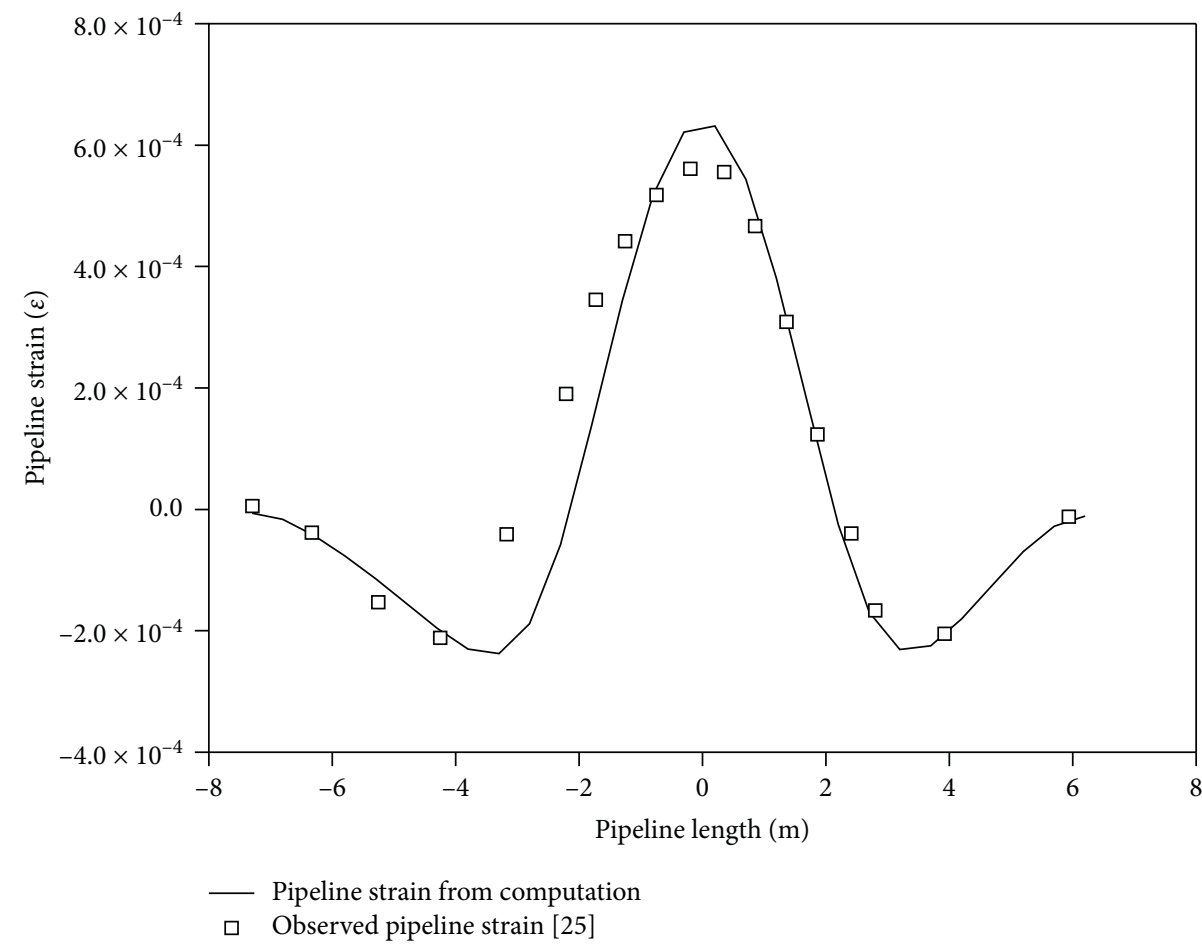

FIGURE 6: Comparison of the pipeline bending strain.

and the cover-to-diameter ratios were 4 and 2, respectively. The responses of pipelines due to twin piggyback tunneling and stacked tunneling were investigated in Test 3 and Test 4, respectively. The soil in the four groups of test uniformly adopted the Toyoura sand, with an internal friction angle of $30^{\circ}$ in the critical state, in which case $K_{0}$ was 0.5 . Table 3 shows the sand parameters after model preparation for the four groups of tests. In all tests, the distances from the 
TABLE 1: Summary of pipeline dimension parameters.

\begin{tabular}{lccccc}
\hline Pipeline dimension & Pipe 1 & Pipe 2 & Pipe 3 & Pipe 4 & Pipe 5 \\
\hline Diameter, $D(\mathrm{~m})$ & 0.27 & 0.61 & 1.02 & 1.51 & 2.01 \\
Wall thickness, $T(\mathrm{~mm})$ & 6 & 10 & 26 & 52 & 95 \\
\hline
\end{tabular}

TABLE 2: Summary of soil parameters.

\begin{tabular}{lccc}
\hline Soil parameter & Loose sand & Medium-density sand & Dense sand \\
\hline Friction angle, $\varphi\left(^{\circ}\right)$ & 30.0 & 35.0 & 40 \\
Bulk unit weight, $\gamma\left(\mathrm{kN} / \mathrm{m}^{3}\right)$ & 16.0 & 17.5 & 19.0 \\
\hline
\end{tabular}

pipeline axis to ground surface were adopted as $5.7525 \mathrm{~m}$, and the mean effective stresses of soil located around the pipeline axis were $59.4 \mathrm{kPa}$ (Test 1 , Test 3, and Test 4) and $59.1 \mathrm{kPa}$ (Test 2). Bolton [26] put forward an empirical formula for calculating the peak internal friction angle of soil:

$$
\begin{aligned}
I_{R} & =D_{r}\left(10-\ln p^{\prime}\right)-1, \\
\varphi_{\max }-\varphi_{c}^{\prime} & =3 I_{R}^{\circ},
\end{aligned}
$$

where $p^{\prime}$ denotes mean effective stress and $D_{r}$ denotes the relative density of soil. In combination with the parameters provided in Table 3, the peak internal friction angles of soil can be calculated as $40.48^{\circ}$ (Test 1 , Test 3 , and Test 4 ) and $40.06^{\circ}$ (Test 2) according to the above equations, respectively. Table 4 provides other calculation parameters in the four centrifuge model tests.

Marshall [23] used centrifuge model tests to explore the effects of tunneling on the internal forces and deformations of pipelines with different stiffness and different volume loss. Table 5 provides the pipeline-related parameters used in the test. These tests were conducted at a centrifuge acceleration of $75 \mathrm{~g}$. Leighton Buzzard Fraction E silica sand was used, whose internal friction angle in the critical state was $32^{\circ}$. After model preparation, the bulk unit weight of the soil was $15.65 \mathrm{kN} / \mathrm{m}^{3}$, and the relative density was $91 \%$. The peak internal friction angle of the soil located around the pipelines was calculated as $45.12^{\circ}$ according to equations (18) and (19). Table 6 provides the parameters for three centrifuge model tests with ground loss rates of $0.5,1.0,2.0$, and $4.0 \%$, respectively.

Figure 10 shows a comparison among calculation results, field measured results, and centrifuge model test results. With the gradual increase of volume loss, the relative pipeline-soil stiffness gradually increases. In other words, the shear strain of soil located around the pipelines increases with increasing volume loss rate. Therefore, the soil stiffness decreases gradually. In this case, by assuming that the pipeline bending stiffness is constant, the relative pipelinesoil stiffness will gradually increase. With the gradual increase of the relative pipeline-soil stiffness, both the relative pipeline-soil curvature and the relative pipeline-soil settlement will gradually decrease. In other words, the mechanical behavior of pipelines will experience a progressive transition from flexible to stiff state. Thus, when analyzing the effects of tunneling on pipelines, we cannot simply apply the greenfield stratum displacement to the pipeline. Instead, reasonable consideration should be given to the effects of the relative pipeline-soil stiffness on calculation results. The prediction range of the proposed calculation formula, used to predict the effects of tunneling on pipeline bending strain and pipeline settlement, can well match field measured results and centrifuge model test results.

\section{Evaluation of the Effect of Tunneling on Adjacent Buried Pipelines}

In the design of municipal pipelines, allowable bending strain $\varepsilon_{\text {pallowable }}$ is commonly adopted to control the service state of pipelines. By assuming that $\varepsilon_{\text {pallowable }}$ equals $800 \mu \varepsilon$ and $400 \mu \varepsilon, k_{g \max }$ can be written as

$$
\kappa_{p \max }=\frac{2}{D} \varepsilon_{\text {pallowable }} .
$$

Substituting it into equation (12), it is obtained that

$$
\frac{\varepsilon_{\text {pallowable }}}{\kappa_{g \max }(D / 2)}=\frac{1}{12.49 x^{0.73}+1} \text {. }
$$

Equation (21) can be written as

$$
\frac{S_{\max }}{i^{2}} \frac{D}{2} \frac{2 \alpha}{n}=\left(12.49 x^{0.73}+1.02\right) \varepsilon_{\text {pallowable }} .
$$

In the same vein, equations (13) and (14) can be written as

$$
\begin{aligned}
& \frac{S_{\max }}{i^{2}} \frac{D}{2} \frac{2 \alpha}{n}=\left(5.78 x^{0.73}+1.02\right) \varepsilon_{\text {pallowable }} \\
& \frac{S_{\max }}{i^{2}} \frac{D}{2} \frac{2 \alpha}{n}=\left(26.11 x^{0.73}+1.02\right) \varepsilon_{\text {pallowable }}
\end{aligned}
$$

Figure 11(a) shows the relationship between the relative pipeline-soil stiffness and the maximum allowable strain of pipelines. When the relative pipeline-soil stiffness is low, pipeline deformation almost superposes with ground deformation. With increasing the relative pipeline-soil stiffness, pipelines experience a gradual transition from flexiblejointed pipelines to stiff-jointed pipelines, resulting in a gradual increase in $\left(S_{\max } / i^{2}\right) \cdot(D / 2) \cdot(2 \alpha / n)$, indicating that, relative to flexible-jointed pipelines, stiff-jointed pipelines can bear a greater ground settlement. When 


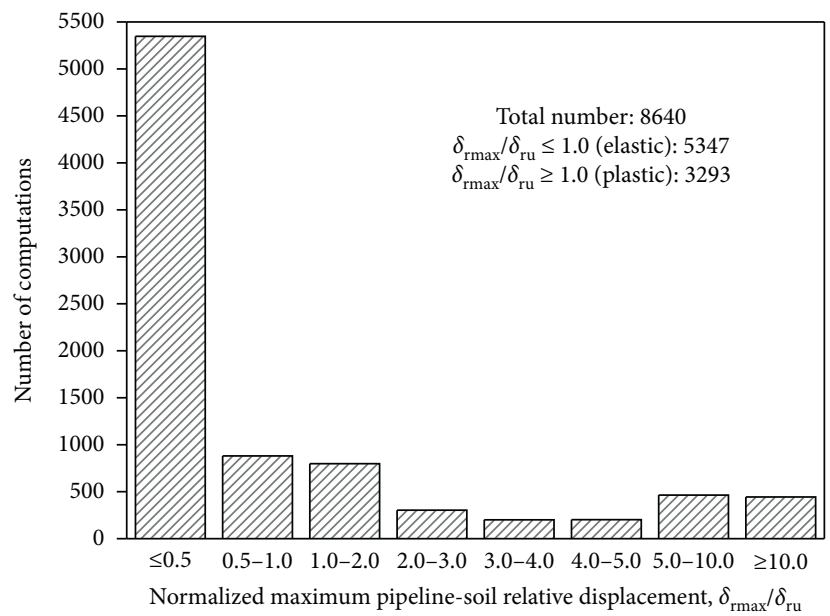

FIGURE 7: Statistical analysis results of maximum relative pipeline-soil displacement.



FIGURE 8: Dimensionless plot of the relative pipeline-soil stiffness versus curvature ratio.

$\varepsilon_{\text {pallowable }}=800 \mu \varepsilon$ (Figure $\left.11(\mathrm{a})\right)$, only one of the test results and field measured results are located in the unsafe zone. When $\varepsilon_{\text {pallowable }}=400 \mu \varepsilon$ (Figure $11(\mathrm{~b})$ ), three groups of data in the test results and field measured results are located in unsafe zone. According to the 12 groups of test results obtained by Marshall [23], with increasing ground loss rate, the service state of pipelines affected by tunneling gradually became unsafe. With other conditions being the same, the stiff-jointed pipelines were safer than the flexible-jointed pipelines. Figure 11 can be directly used to evaluate the effects of single and twin tunneling on the safety of existing buried pipelines when the following parameters are provided: basic pipeline parameters ( $D$ and $T$ ), pipeline material and burial depth $(E$ and $H)$, basic soil parameters $(\gamma$ and $\varphi)$, and curve parameters of ground settlement due to tunneling $\left(S_{\max }, i\right.$, and $\left.\alpha\right)$. 




- Calculation results (total no. 8640) — Upper $90 \%$ prediction interval

- Best-fit curve _ Lower $90 \%$ prediction interval

FIgURE 9: Dimensionless plot of the relative pipeline-soil stiffness versus settlement ratio.

TABle 3: Parameters of sand layer materials after model preparation in the four centrifuge model tests.

\begin{tabular}{lccc}
\hline Test identity & Initial void ratio $(e)$ & Density, $\rho\left(\mathrm{kg} / \mathrm{m}^{3}\right)$ & Initial relative density, $D_{r}(\%)$ \\
\hline Test 1 & 0.711 & 1.549 & 70 \\
Test 2 & 0.719 & 1.542 & 68 \\
Test 3 & 0.711 & 1.549 & 70 \\
Test 4 & 0.711 & 1.549 & 70 \\
\hline
\end{tabular}

TABLE 4: Summary of the calculation parameters of the four centrifuge model tests.

\begin{tabular}{|c|c|c|c|c|c|c|c|c|}
\hline Test identity & $\begin{array}{c}S_{\max } \\
(\mathrm{mm})\end{array}$ & $i(\mathrm{~m})$ & $\alpha$ & $\begin{array}{l}\kappa_{p \max } / \\
k_{g \max }\end{array}$ & $\begin{array}{c}K_{u} \\
(\mathrm{MPa})\end{array}$ & $\begin{array}{c}K_{d} \\
(\mathrm{MPa})\end{array}$ & {$\left[E_{P} I_{P} /\left(K_{u}^{0.9} K_{u}^{0.1}\left((n / 2 \alpha)^{0.5} i\right)^{4}\right)\right]\left[S_{\max } /(n / 2 \alpha)^{0.5} i\right]^{0.25}$} & $\begin{array}{l}S_{p \max } \\
S_{g \text { max }}\end{array}$ \\
\hline $\begin{array}{l}\text { Test } 1 \text { (single } \\
\text { tunnel) }\end{array}$ & 35.0 & 7.94 & 0.667 & 0.253 & 8.22 & 80.21 & 0.135 & 0.669 \\
\hline $\begin{array}{l}\text { Test } 1 \text { (twin } \\
\text { tunnels) }\end{array}$ & 52.9 & 9.02 & 0.524 & 0.265 & 8.08 & 73.84 & 0.081 & 0.832 \\
\hline $\begin{array}{l}\text { Test } 2 \text { (single } \\
\text { tunnel) }\end{array}$ & 50.3 & 6.01 & 0.690 & 0.131 & 8.22 & 80.21 & 0.500 & 0.695 \\
\hline $\begin{array}{l}\text { Test } 2 \text { (twin } \\
\text { tunnels) }\end{array}$ & 89.4 & 8.26 & 0.675 & 0.161 & 8.22 & 80.21 & 0.148 & 0.674 \\
\hline $\begin{array}{l}\text { Test } 3 \text { (twin } \\
\text { tunnels) }\end{array}$ & 67.3 & 7.65 & 0.519 & 0.242 & 8.22 & 80.21 & 0.087 & 0.773 \\
\hline $\begin{array}{l}\text { Test } 4 \text { (twin } \\
\text { tunnels) }\end{array}$ & 70.3 & 7.43 & 0.426 & 0.176 & 8.22 & 80.21 & 0.183 & 0.691 \\
\hline
\end{tabular}

Note. Test 1 (single tunnel) means that the first tunneling has been finished in the first centrifuge model test; Test 1 (twin tunnels) means that the twin tunneling has been completed in the first centrifuge model test. This interpretive mode also applies to the other test identities. 
TABle 5: Pipeline parameters used in the centrifuge model tests.

\begin{tabular}{lcccccc}
\hline Pipeline & Material & Section shape & $\begin{array}{c}\text { Section width/ } \\
\text { diameter }(\mathrm{mm})\end{array}$ & Wall thickness $(\mathrm{mm})$ & Elastic modulus, $E_{p}(\mathrm{MPa})$ & Stiffness, $E_{p} I_{p}\left(\mathrm{~N} \cdot \mathrm{m}^{2}\right)$ \\
\hline Pipe 1 & Aluminium pipeline & Square & 19.05 & 1.63 & 70 & 70 \\
Pipe 2 & Aluminium pipeline & Tubular & 19.06 & 6.23 & $2.56 \times 10^{10}$ \\
Pipe 3 & Plastic pipeline & Tubular & 16.00 & 0.26 & 2.9 & $7.54 \times 10^{9}$ \\
\hline
\end{tabular}

TABLE 6: Summary of the calculation parameters of the centrifuge model tests.

\begin{tabular}{|c|c|c|c|c|c|c|c|c|}
\hline Test identity & $\begin{array}{c}S_{\max } \\
(\mathrm{mm})\end{array}$ & $i(\mathrm{~m})$ & $\alpha$ & $\begin{array}{l}\kappa_{p \max } / \\
k_{g \max }\end{array}$ & $\begin{array}{c}K_{u} \\
(\mathrm{MPa})\end{array}$ & $\begin{array}{c}K_{d} \\
(\mathrm{MPa})\end{array}$ & {$\left[E_{P} I_{P} /\left(K_{u}^{0.9} K_{d}^{0.1}\left((n / 2 \alpha)^{0.5} i\right)^{4}\right)\right]\left[S_{\max } /(n / 2 \alpha)^{0.5} i\right]^{0.25}$} & $\begin{array}{l}S_{p \max } / \\
S_{g \max }\end{array}$ \\
\hline $\begin{array}{l}\text { TunPipe1 } \\
(0.5 \%)\end{array}$ & 5.5 & 7.75 & 0.587 & 0.304 & 17.76 & 149.82 & 0.05560 & 0.78429 \\
\hline $\begin{array}{l}\text { TunPipe1 } \\
(1.0 \%)\end{array}$ & 13.3 & 5.50 & 0.311 & 0.224 & 17.76 & 149.82 & 0.23228 & 0.57485 \\
\hline $\begin{array}{l}\text { TunPipe1 } \\
(2.0 \%)\end{array}$ & 25.0 & 4.39 & 0.066 & 0.143 & 17.76 & 149.82 & 0.44059 & 0.48315 \\
\hline $\begin{array}{l}\text { TunPipe1 } \\
(4.0 \%)\end{array}$ & 32.8 & 3.80 & 0.051 & 0.090 & 17.76 & 149.82 & 0.84092 & 0.34895 \\
\hline $\begin{array}{l}\text { TunPipe2 } \\
(0.5 \%)\end{array}$ & 5.5 & 7.75 & 0.587 & 0.432 & 16.80 & 143.40 & 0.01729 & 0.88339 \\
\hline $\begin{array}{l}\text { TunPipe2 } \\
(1.0 \%)\end{array}$ & 13.3 & 5.50 & 0.311 & 0.365 & 16.80 & 143.40 & 0.03552 & 0.82340 \\
\hline $\begin{array}{l}\text { TunPipe2 } \\
(2.0 \%)\end{array}$ & 25.0 & 4.39 & 0.066 & 0.234 & 16.80 & 143.40 & 0.13702 & 0.62913 \\
\hline $\begin{array}{l}\text { TunPipe2 } \\
(4.0 \%)\end{array}$ & 32.8 & 3.80 & 0.051 & 0.196 & 16.80 & 143.40 & 0.26152 & 0.52575 \\
\hline $\begin{array}{l}\text { TunPipe3 } \\
(0.5 \%)\end{array}$ & 5.5 & 7.75 & 0.587 & 0.940 & 17.28 & 141.67 & $4.56631 E-4$ & 0.99594 \\
\hline $\begin{array}{l}\text { TunPipe3 } \\
(1.0 \%)\end{array}$ & 13.3 & 5.50 & 0.311 & 0.900 & 17.28 & 141.67 & $9.37974 E-4$ & 0.98096 \\
\hline $\begin{array}{l}\text { TunPipe3 } \\
(2.0 \%)\end{array}$ & 25.0 & 4.39 & 0.066 & 0.785 & 17.28 & 141.67 & 0.00362 & 0.96090 \\
\hline $\begin{array}{l}\text { TunPipe3 } \\
(4.0 \%)\end{array}$ & 32.8 & 3.80 & 0.051 & 0.711 & 17.28 & 141.67 & 0.00691 & 0.95091 \\
\hline
\end{tabular}

Note. TunPipe1 (0.5\%) means that the pipeline in the centrifuge model test adopts the square aluminum pipeline provided in Table 5 and that the ground loss rate caused by tunneling is $0.5 \%$. This interpretive mode also applies to other test identities. 




(a)

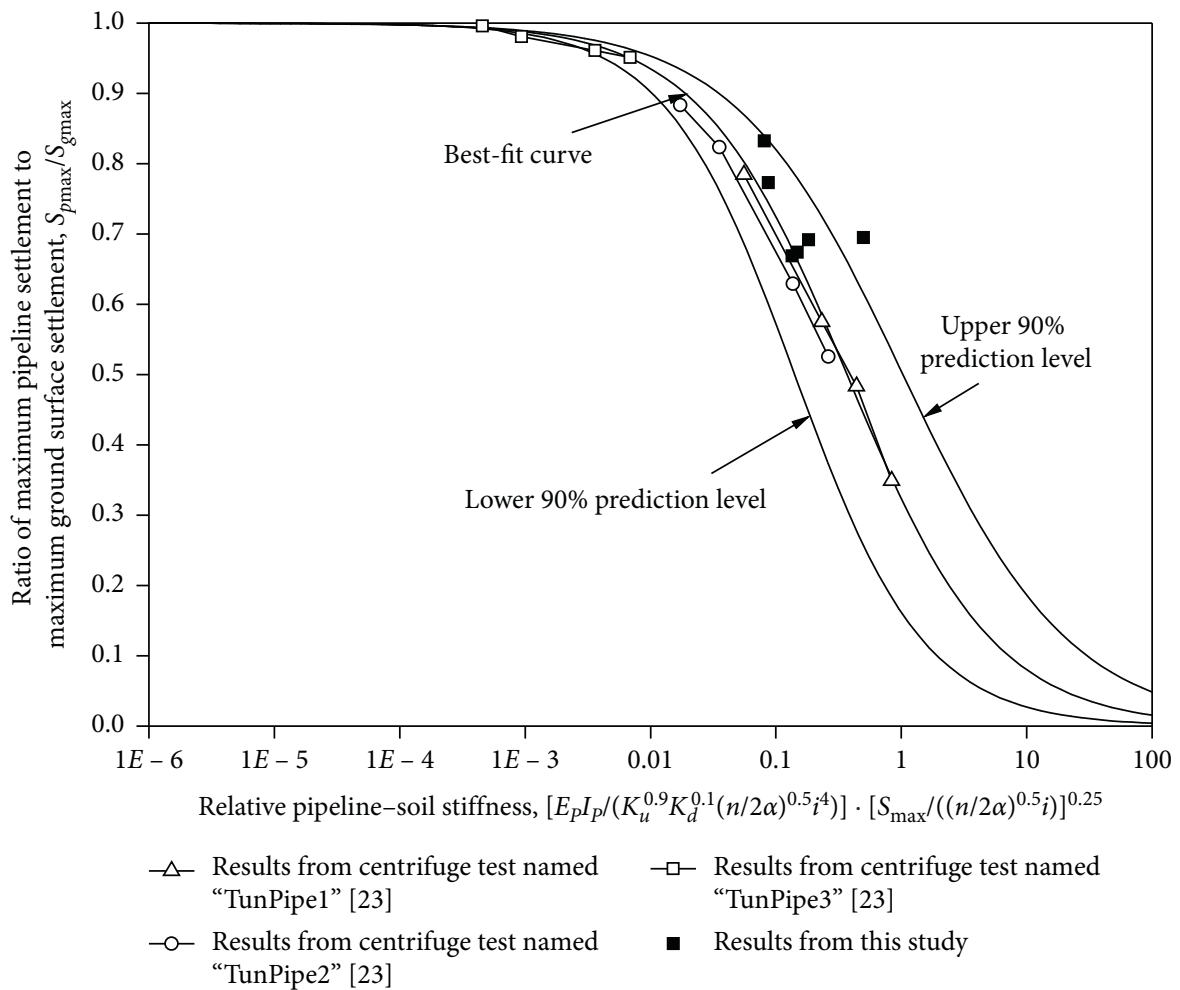

(b)

FIGURE 10: Comparison of calculation results with field and centrifuge test results: (a) relative pipeline-soil stiffness and ratio of maximum pipe curvature to maximum ground curvature; (b) relative pipeline-soil stiffness and ratio of maximum pipeline settlement to maximum ground settlement. 


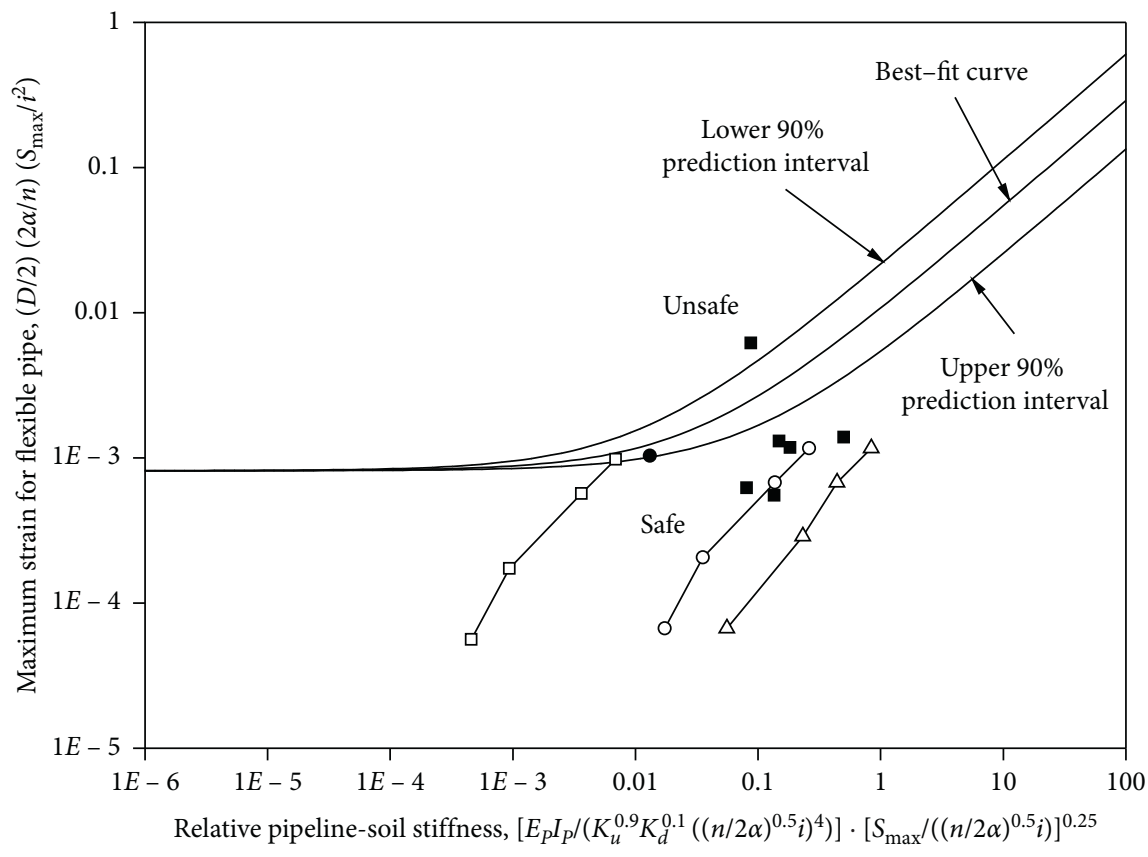

$-\triangle$ Results from centrifuge test named

- Results from field test [25] "TunPipe1" [23]

$-\circ$ Results from centrifuge test named “TunPipe2" [23]

- Results from this study

$\rightarrow \square$ Results from centrifuge test named "TunPipe3" [23]

(a)

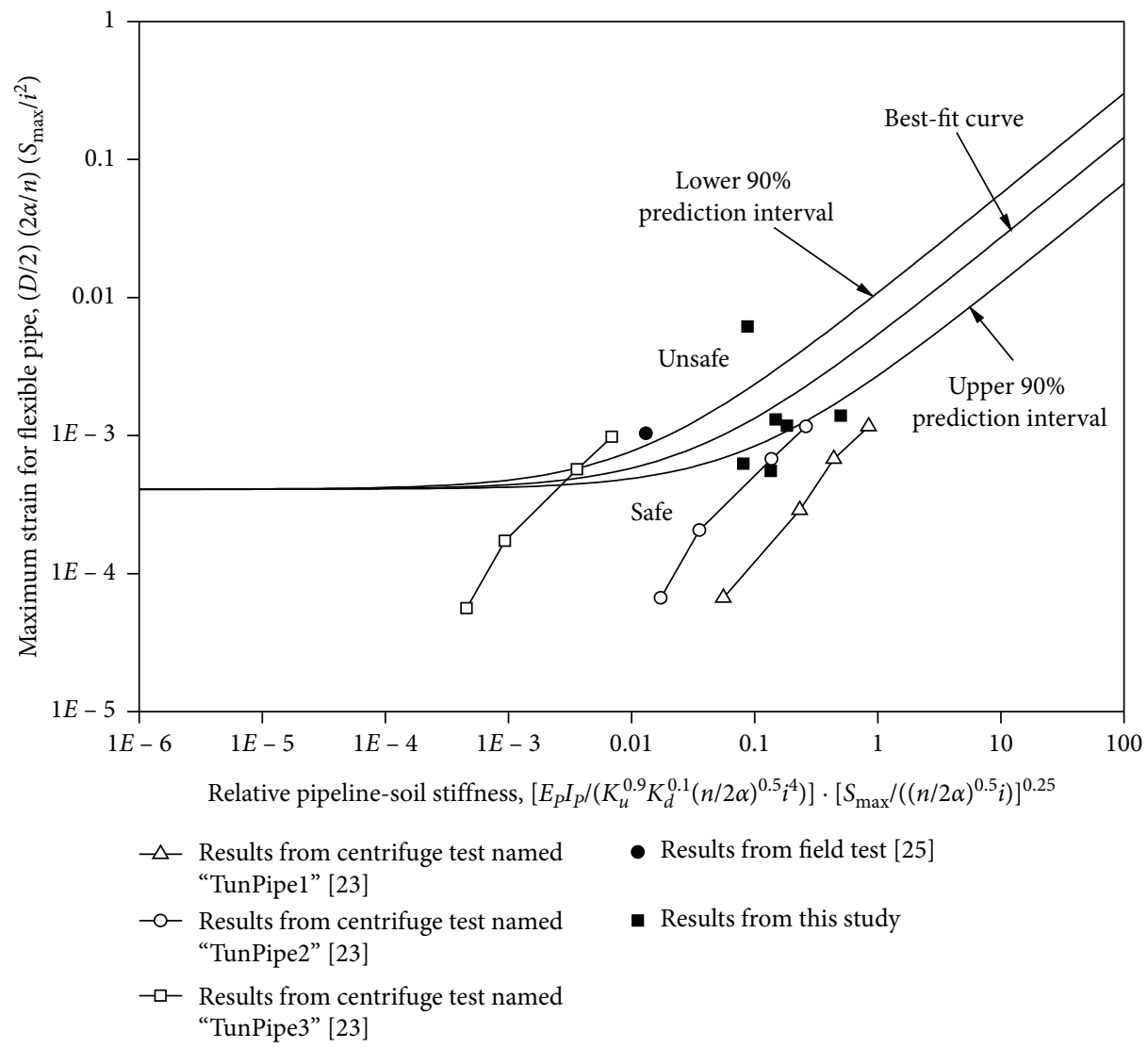

(b)

FIGURE 11: Illustration of pipeline safety criteria due to tunneling: (a) $\varepsilon_{\text {pallowable }}=800 \mu \varepsilon$; (b) $\varepsilon_{\text {pallowable }}=400 \mu \varepsilon$. 


\section{Conclusions}

(1) When analyzing the effects of tunneling on existing buried pipelines, it is undesirable to simply apply greenfield stratum displacement. Instead, a consideration should be given to the effects of the relative pipeline-soil stiffness on calculation results.

(2) The expression for the relative pipeline-soil stiffness proposed in this paper correlates well with the relative pipeline-soil curvature and relative pipelinesoil settlement. When relative pipeline-soil stiffness is lower than $10^{-4}$, the mechanical behavior of pipelines is similar to that of flexible-jointed pipelines. With increasing the relative pipeline-soil stiffness, the mechanical behavior of pipelines gradually is stiffened. When the relative pipeline-soil stiffness is larger than 10 , the relative pipeline curvature $\left(\kappa_{p \max } / k_{g \text { max }}\right)$ and relative pipeline settlement $\left(S_{p \max } / S_{g \text { max }}\right)$ gradually approach to 0 , meaning that the pipeline displacement is affected by stratum displacement to a smaller extent.

(3) Based on the calculation method proposed in this paper, the maximum bending strain and maximum settlement of pipelines can be calculated precisely when the basic pipeline parameters, pipeline material and burial depth, basic soil parameters, and curve parameters of ground settlement due to tunneling are provided.

(4) The proposed equation for the relative pipeline-soil stiffness and relative pipeline curvature and that for the relative pipeline-soil stiffness and relative pipeline settlement can be used not only to predict the maximum bending strain and maximum settlement of pipelines caused by single and twin tunneling, but also to evaluate the effects of single and twin tunneling on the safety of existing buried pipelines.

\section{Data Availability}

The data used to support the findings of this study are included within the article.

\section{Conflicts of Interest}

The authors declare that they have no conflicts of interest.

\section{Acknowledgments}

The authors would like to acknowledge the financial support of the China Postdoctoral Science Foundation (no. 2019M663874XB), Special Fund for Science and Technology Bases and Talented Young Persons in Guangxi (no. GuiKe AD20238069), and Key Project of Natural Science Foundation of Guangxi (no. 2020GXNSFDA238024).

\section{References}

[1] H.-N. Wu, S.-L. Shen, S.-M. Liao, and Z.-Y. Yin, "Longitudinal structural modelling of shield tunnels considering shearing dislocation between segmental rings," Tunnelling and Underground Space Technology, vol. 50, pp. 317-323, 2015.

[2] H.-N. Wu, S.-L. Shen, J. Yang, and A. Zhou, "Soil-tunnel interaction modelling for shield tunnels considering shearing dislocation in longitudinal joints," Tunnelling and Underground Space Technology, vol. 78, pp. 168-177, 2018.

[3] H.-N. Wu, S.-L. Shen, R.-P. Chen, and A. Zhou, "Threedimensional numerical modelling on localised leakage in segmental lining of shield tunnels," Computers and Geotechnics, vol. 122, no. 1-12, Article ID 103549, 2020.

[4] A. Klar, T. E. B. Vorster, K. Soga, and R. J. Mair, "Soil-pipe interaction due to tunnelling: comparison between Winkler and elastic continuum solutions," Géotechnique, vol. 55, no. 6, pp. 461-466, 2005.

[5] P. B. Attewell, J. Yeates, and A. R. Selby, Soil Movements Induced by Tunneling and Their Effects on Pipelines and Structures, Blackie \& Son, London, UK, 1986.

[6] J. Yu, C. Zhang, and M. Huang, "Soil-pipe interaction due to tunnelling: assessment of Winkler modulus for underground pipelines," Computers and Geotechnics, vol. 50, pp. 17-28, 2013.

[7] A. Klar and A. M. Marshall, "Linear elastic tunnel pipeline interaction: the existence and consequence of volume loss equality," Géotechnique, vol. 65, no. 9, pp. 788-792, 2015.

[8] Y. Wang, J. Shi, and C. W. W. Ng, "Numerical modeling of tunneling effect on buried pipelines," Canadian Geotechnical Journal, vol. 48, no. 7, pp. 1125-1137, 2011.

[9] J. W. Shi and L. Chen, "Investigation of bending deformation of underground pipelines induced by differential ground movements," Rock and Soil Mechanicas, vol. 38, no. 4, pp. 1164-1170, 2017.

[10] T. E. Vorster, A. Klar, K. Soga, and R. J. Mair, "Estimating the effects of tunneling on existing pipelines," Journal of Geotechnical and Geoenvironmental Engineering, vol. 131, no. 11, pp. 1399-1410, 2005.

[11] A. M. Marshall, A. Klar, and R. J. Mair, “Tunneling beneath buried pipes: view of soil strain and its effect on pipeline behavior," Journal of Geotechnical and Geoenvironmental Engineering, vol. 136, no. 12, pp. 1664-1672, 2010.

[12] A. Klar, I. Elkayam, and A. M. Marshall, "Design oriented linear-equivalent approach for evaluating the effect of tunneling on pipelines," Journal of Geotechnical and Geoenvironmental Engineering, vol. 142, no. 1, pp. 1-8, Article ID 04015062, 2015.

[13] X. Shi, C. Rong, H. Cheng, L. Cui, and J. Kong, "An energy solution for predicting buried pipeline response induced by tunneling based on a uniform ground movement model," Mathematical Problems in Engineering, vol. 2020, Article ID 7905750, 12 pages, 2020.

[14] Z. Zhang, M. Zhang, Q. Zhao, L. Fang, Z. Ding, and M. Shi, "Interaction analyses between existing pipeline and quasirectangular tunneling in clays," KSCE Journal of Civil Engineering, vol. 25, no. 1, pp. 326-344, 2020.

[15] M. Huang, X. Zhou, J. Yu, C. F. Leung, and J. Q. W. Tan, "Estimating the effects of tunnelling on existing jointed pipelines based on Winkler model," Tunnelling and Underground Space Technology, vol. 86, pp. 89-99, 2019.

[16] M. Saiyar, P. Ni, W. A. Take, and I. D. Moore, "Response of pipelines of differing flexural stiffness to normal faulting," Géotechnique, vol. 66, no. 4, pp. 275-286, 2016.

[17] G. Wei, S. Hu, S. Hu, J. J. Xing, and Q. Ye, "Effect of double line parallel shield excavation on A djacent underground 
pipelines," Journal of Engineering Science and Technology Review, vol. 9, no. 1, pp. 167-173, 2016.

[18] S. Ma, Y. Shao, Y. Liu, J. Jiang, and X. Fan, "Responses of pipeline to side-by-side twin tunnelling at different depths: 3D centrifuge tests and numerical modelling," Tunnelling and Underground Space Technology, vol. 66, pp. 157-173, 2017.

[19] S. K. Ma, Y. Liu, Y. Shao, Z. B. Duan, and H. B. Lv, "Effects of twin shield tunneling with different construction sequences and different relative locations on adjacent pipelines," Chinese Journal of Geotechnical Engineering, vol. 36, no. 10, pp. 1159-1166, 2017.

[20] A. M. Marshall, R. Farrell, A. Klar, and R. Mair, "Tunnels in sands: the effect of size, depth and volume loss on greenfield displacements," Géotechnique, vol. 62, no. 5, pp. 385-399, 2012.

[21] S. Suwansawat and H. H. Einstein, "Describing settlement troughs over twin tunnels using a superposition technique," Journal of Geotechnical and Geoenvironmental Engineering, vol. 133, no. 4, pp. 445-468, 2007.

[22] T. E. B. Vorster, Effects of Tunneling on Buried Pipes, University of Cambridge, Cambridge, UK, 2005.

[23] A. Marshall, Tunneling in Sand and its Effect on Pipelines and Piles, University of Cambridge, Cambridge, UK, 2009.

[24] Committee on Gas and Liquid Fuel Lifeline, Guidelines for the Seismic Design of Oil and Gas Pipeline Systems, American Society of Civil Engineers, New York, NY, USA, 1984.

[25] N. Takagi, K. Shimamura, and N. Nishio, "Buried pipe response to adjacent ground movements associated with tunneling and excavations," in Proceedings of the $3 \mathrm{rd}$ International Conference on Ground Movements and Structures, Pentech Press, Cardiff, Wales, July 1984.

[26] M. D. Bolton, "The strength and dilatancy of sands," Géotechnique, vol. 36, no. 1, pp. 65-78, 1986. 